\title{
Muscle activity during backward perturbation response in patients with clinical vertebral compression fractures
}

\author{
Tomoya Kishimoto ${ }^{1,2 *}$, Kastushi Kuniyasu ${ }^{3}$, Tadanobu Suehiro ${ }^{4}$, Kenichi Kobara ${ }^{4}$ \\ 'Department of Rehabilitation, Watanabe Hospital, Niimi, Japan \\ ${ }^{2}$ Graduate School of Health Science and Technology, Kawasaki University of Medical Welfare, Kurashiki, Japan \\ ${ }^{3}$ Department of Physical Therapy, Faculty of Rehabilitation, Kawasaki University of Medical Welfare, Kurashiki, Japan \\ ${ }^{4}$ Department of Rehabilitation, Faculty of Health Science and Technology, Kawasaki University of Medical Welfare, Kurashiki, Japan
}

The present study examined muscle activity in response to backward perturbation in patients with clinical vertebral compression fracture (CVCF). The subjects were 32 patients aged 65 years and above consisting of 16 each with (CVCF group) and without (control group) CVCF. The time to peak activity, and time of onset of muscle activity of the anterior tibial, vastus medialis, and rectus abdominis muscles when unexpected backward perturbation was applied were evaluated by surface electromyography. The strength of perturbation was $4 \%$ or $6 \%$ of the subject's body weight. In addition, the presence of the stepping reaction to perturbation, severity of low back pain, and vertebral alignment were evaluated. Each item was compared between the two groups. In the
CVCF group, kyphosis and severity of low back pain were significantly more severe, the time to peak activity of the anterior tibial muscle after the application of perturbation at $6 \%$ of the body weight was significantly shorter, and the time of onset of activity of the rectus abdominis muscle was significantly delayed. This suggests that the time to peak activity of the anterior tibial muscle is shortened and the time of onset of activity of the rectus abdominis muscle is delayed in unexpected backward perturbation in patients with CVCF.

Keywords: Vertebral compression fracture, Muscle activity, Backward perturbation

\section{INTRODUCTION}

Vertebral compression fractures are the osteoporotic fracture most frequently observed in older individuals (Freitas et al., 2008). As characteristics of vertebral compression fractures, it has been reported that an existing vertebral compression fracture itself is a risk factor for new vertebral compression fractures (Klotzbuecher et al., 2000), and that the walking ability, balancing ability, and activities of daily living (ADL) decline with increases in the number of fractured vertebrae (Arima et al., 2017; O’Neill et al., 2004). The number of fractured vertebrae has also been demonstrated to affect survival (Hasserius et al., 2003; Ismail et al., 1998). Twothirds of vertebral compression fractures are asymptomatic or mildly symptomatic and may remain unrecognized (Cummings et al., 1998). If vertebral compression is confirmed later by imag- ing examination, it is termed morphometric fracture and treatment for osteoporosis is performed. On the other hand, if a patient consults a medical facility due to symptoms, such as low back pain, the condition is termed "clinical fracture" and is treated as fracture (Freitas et al., 2008). The incidence of clinical vertebral compression fracture (CVCF) is low, comprising 22\%-33\% of all vertebral compression fractures (Cummings et al., 1998), but it is internationally higher than that of proximal femoral fracture (Bow et al., 2012). Moreover, the cause of CVCF is mild trauma associated with events, such as falling, in approximately half of the patients (Freitas et al., 2008). Therefore, it is important to prevent secondary fracture due to falling after CVCF.

The direction of fall of older individuals has been reported to be forward in 44\%, backward in 41\%, and sideways in 37\% (Crenshaw et al., 2017). However, in the same report, protective reac-
${ }^{*}$ Corresponding author: Tomoya Kishimoto (i) https://orcid.org/0000-0001-7674-4194 Department of Rehabilitation, Watanabe Hospital, 2278-1 Takao, Niimi 718-0003, Japan

E-mail: abeta09220507@gmail.com

Received: August 27, 2019 / Accepted: September 15, 2019
This is an Open Access article distributed under the terms of the Creative Commons Attribution Non-Commercial License (http://creativecommons.org/licenses/by-nc/4.0/) which permits unrestricted non-commercial use, distribution, and reproduction in any medium, provided the original work is properly cited. 
tions, such as stepping, were observed in $82 \%$ of the forward falls but in only $37 \%$ of the backward falls. In addition, the impact on the body due to falling was large in the wrist and hand in forward falls, but in the hip joint and pelvis in backward falls. Fracture of the spine or lower limbs causes a more marked decline in ADL than fracture of the upper limbs (Stone et al., 2003). Therefore, after falls by older individuals, it is important to analyze posture control of the back in consideration of the difficulty in recovery after falling and sites that sustain impact even though there is no difference in the frequency of the direction of falls. Horak and Nashner (1986) suggested that muscle responses contribute to recovery of stability when balance is impaired by perturbation. Therefore, evaluation of the characteristics of muscle activity during perturbation response is considered to aid in the formulation of programs to prevent falls and rehabilitate CVCF patients. We therefore examined muscle activity during the backward perturbation response in CVCF patients.

\section{MATERIALS AND METHODS}

\section{Materials}

The subjects were 16 patients with CVCF (CVCF group) and 16 non-CVCF patients (control group). This is a cross-sectional study comparing these two groups. Women aged 65 years or older who visited or were admitted for examination to the authors' institution between May and July 2019, lived independently at home, and were able to walk without walking aids were enrolled. Patients who had marked visual impairment or sensory impairment of the lower limbs, those with impairment of vestibular function, those with neuromuscular disorders, such as multiple sclerosis and multiple myositis, those with a history of cerebrovascular disorders or orthopedic disorders of the lower limbs, those who complained of severe pain in the standing position or during walking, and those who did not understand or consent to the study were excluded. In addition, those within 6 months from the diagnosis of vertebral compression fracture and those with level 4 or greater low back pain by the numerical rating scale (NRS) were excluded from the CVCF group. Patients who had lumbar pain within 2 years prior to the study were excluded from the control group. CVCF patients were defined as patients diagnosed with vertebral compression fracture complaining primarily of low back pain who were treated conservatively by corseting. Before data collection, all the procedures were explained to the participants, and they signed an informed consent form. This study was carried out after approval by the Institutional Review Board of Medical
Corporation Shiseikai Watanabe Hospital (approval number: 01703).

\section{Methods}

For the attributes of the subjects, their age, height, body weight, body mass index (BMI), disease primarily responsible for hospital visits or hospitalization, fractured vertebral bodies, number of compression fractures, and circumstances of compression fracture were extracted. The evaluation items were muscle activity and the presence of stepping reaction when backward perturbation was applied, severity of low back pain, and vertebral alignment. The disease primarily responsible for hospital visits or hospitalization, fractured vertebral bodies, number of compression fractures, and circumstances of compression fracture were extracted from electronic charts. The most severe pain felt in daily living orally expressed by the patients according to an NRS was recorded as the severity of low back pain.

Backward perturbation for the measurement of muscle activity was implemented by the postural stress test according to the report by Chandler et al. (1990). Via this method, backward perturbation is applied by winding a belt with a rope around the waist of the standing subject, hanging a weight on the other side of the rope, and allowing the weight to fall freely via a pulley. The setting of measurement is shown in Fig. 1. In this study, a similar measurement condition was prepared by attaching a pulley to a height-adjustable bed. The weight was placed on a special table (free-fall device) set at the highest level not causing tension in the rope, and the measurer was able to freely decide the timing of the fall. The subjects stood with their eyes open and both arms hanging along the sides of the trunk, and legs comfortably spread at 60 $\mathrm{cm}$ from the bed with their back turned to it. They were instructed to maintain a relaxed standing position. A nonstretchable band of $10 \mathrm{~cm}$ in width and $150 \mathrm{~cm}$ in length was tightly wound around the subject's pelvis by positioning the upper margin of the band at the level of the cephalic end of the iliac crest. Regarding the procedure, before measurement, the subjects were uniformly instructed, "Your body will be pulled backwards, but please try not to fall and keep standing." There was no practice before the measurement. The measurer was positioned between the subject and the bed to prevent the subject from falling. Five seconds after the above instruction was given, the weight was allowed to fall, and backward perturbation was applied to the subject. Although the objective of this study was to observe muscle activity in response to unexpected perturbation, the minimum necessary explanation was given as above concerning the content of the test in 


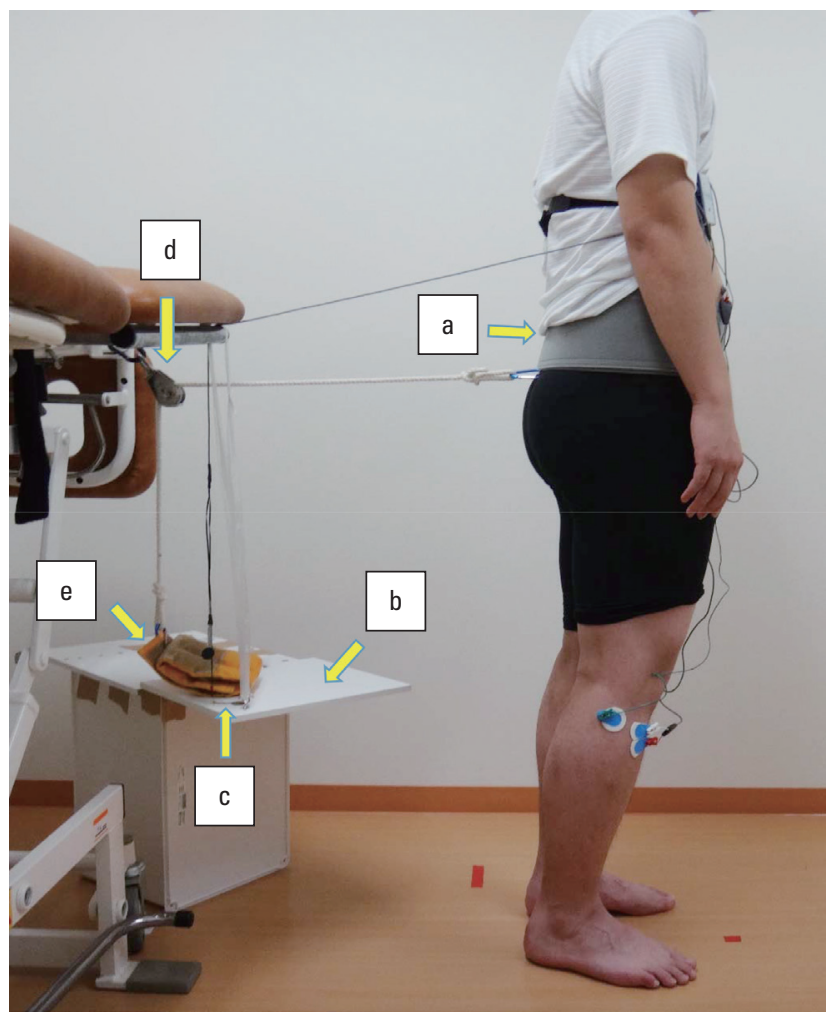

Fig. 1. The setting of measurement for backward perturbation response $(a$, winding belt; $b$, free-fall device; $c$, foot switch; $d$, pulley; e, weight). Backward perturbation is applied by winding a belt with a rope around the waist of the standing subject, hanging a weight on the other side of the rope, and allowing the weight to fall freely via a pulley.

consideration of ethics. The measurements were performed under two conditions: using a weight equaling $4 \%$ of the body weight and a weight equaling $6 \%$ of the body weight $(4 \% \mathrm{BW}$ and $6 \% \mathrm{BW}$ conditions, respectively). The weight of the weights was rounded to the nearest $100 \mathrm{~g}$. Measurement was performed once each under the $4 \% \mathrm{BW}$ and $6 \% \mathrm{BW}$ conditions in this order. At the same time, the presence of backward stepping was visually assessed by the measurer. Stepping was judged to be absent if the subject was able to remain standing in the original position and present if the subject stepped backward with either foot.

Muscle activity was measured using a surface electromyography at a sampling frequency of 1,000 Hz. The surface electrodes were attached at the right anterior tibial muscle (three fingerbreadths inferomedially from the fibular head), vastus medialis muscle (two fingerbreadths inferomedially from the upper margin of the patella), and rectus abdominis muscle $(2-3 \mathrm{~cm}$ laterally from the umbilicus), and the reference electrode was placed at the right fibular head. The interelectrode distance was $2.5 \mathrm{~cm}$. The time of falling of the weight was determined with a foot switch attached to the testing device, on which the weight was placed. The electromyograms obtained were processed using band-pass filters (10-500 $\mathrm{Hz}$ ) and full-wave rectified. From the processed electromyograms, the time to peak activity and time of onset of activity of each muscle were calculated. The time to peak activity was defined as the time from the fall of the weight to peak activity of each muscle. The time of onset of activity was the time from the fall of the weight to the onset of activity of each muscle. The onset of muscle activity was defined as the time when the amplitude of the activity exceeded two standard deviations from the mean activity during relaxed standing.

The vertebral alignment was evaluated using the kyphosis index representing the degree of spinal kyphosis according to the report by Milne and Lauder (1974). During the measurement, the subjects comfortably sat on the bed with their arms crossed and plantae separated from the floor. The subjects wore only underwear, a 50-cm flexible ruler was applied to the dorsal curvature from the spinous process of the 7 th cervical vertebra $(\mathrm{C} 7)$ to the spinous process of the 4th lumbar vertebra (L4), and the shape of the ruler was traced on paper. The line from $\mathrm{C} 7$ to $\mathrm{L} 4$ on the trace was defined as $\mathrm{L}(\mathrm{cm})$, the distance from line $\mathrm{L}$ to the apex of the curvature was defined as $\mathrm{H}(\mathrm{cm})$, and the kyphosis index was calculated as the percentage of $\mathrm{H}$ relative to $\mathrm{L}(\mathrm{H} / \mathrm{L} \times 100)$ using the equation by Milne and Lauder (1974). The measurement was performed 3 times, and the mean of the three measurements was used for analysis.

Regarding the attributes of the subjects and evaluation items, the normality of age, height, body weight, BMI, time to peak activity, time of onset of muscle activity, and kyphosis index were examined by the Shapiro-Wilk test. Based on the results of the Shapiro-Wilk test, the $t$-test was performed for items that exhibited normal distribution, and the Mann-Whitney $U$-test was performed for those that did not for comparison between the two groups. Pain expressed using the NRS was compared by the Mann-Whitney $U$-test, and the presence of stepping was compared by the chi-square test between the two groups. IBM SPSS Statistics ver. 21.0 (IBM Co., Armonk, NY, USA) was used for statistical analysis. All tests were performed at the significance level of $5 \%$.

\section{RESULTS}

The attributes, kyphosis index, NRS score, and presence of stepping between the CVCF and control groups are compared in Table 1. No significant difference was observed in age, height, body 
Table 1. Attributes, kyphosis index, NRS score, and presence of stepping between the CVCF and control groups

\begin{tabular}{|c|c|c|c|}
\hline Variable & $\begin{array}{c}\text { CVCF } \\
(n=16)\end{array}$ & $\begin{array}{l}\text { Control } \\
(n=16)\end{array}$ & $P$-value \\
\hline Age (yr) & $79.5 \pm 6.6$ & $75.7 \pm 6.0$ & $0.10^{\mathrm{a})}$ \\
\hline Height (cm) & $146.1 \pm 7.0$ & $146.7 \pm 4.9$ & $0.79^{\mathrm{al}}$ \\
\hline Weight (kg) & $47.1 \pm 7.1$ & $49.2 \pm 6.2$ & $0.38^{\mathrm{a})}$ \\
\hline Body mass index (kg/m²) & $22.0 \pm 2.5$ & $22.9 \pm 2.7$ & $0.34^{\mathrm{al}}$ \\
\hline \multicolumn{4}{|l|}{ Fractured vertebral bodies } \\
\hline Thoracic vertebrae:lumbar vertebrae & $5: 11$ & - & - \\
\hline \multicolumn{4}{|l|}{ No. of compression fractures } \\
\hline Singular:multiple & $2: 14$ & - & - \\
\hline Kyphosis index & $14.4 \pm 4.0$ & $9.6 \pm 1.9$ & $0.00^{\mathrm{a})}$ \\
\hline NRS & $0.5(0-3.0)$ & 0 & $0.02^{b)}$ \\
\hline \multicolumn{4}{|l|}{ Stepping reaction } \\
\hline 4\%BW (positive:negative) & $11: 5$ & $13: 3$ & $0.34^{c \mid}$ \\
\hline 6\%BW (positive:negative) & $7: 9$ & $8: 8$ & $1.00^{c)}$ \\
\hline
\end{tabular}

Values are presented as mean \pm standard deviation, number, or median (interquartile range).

NRS, numerical rating score; CVCF, clinical vertebral compression fracture; BW, body weight.

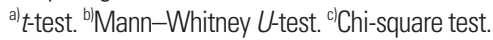

weight, BMI, or presence of stepping between the two groups. The kyphosis index $(P<0.01)$ and NRS score $(P<0.05)$ were significantly higher in the CVCF group than in the control group. The primary reason for hospital visits or hospitalization was follow-up after vertebral compression fracture in 7 , hypertension in 4, hospitalization for examination associated with gastrointestinal endoscopy in 2, dyslipidemia in 1, osteoporosis in 1, and bronchitis in 1 in the CVCF group, and osteoporosis in 4, hospitalization for examination associated with gastrointestinal endoscopy in 4, hypertension in 2, scapulohumeral periarthritis in 2, finger fracture in 2, follow-up after pneumonia in 1 , and treatment for left lower leg contusion in 1 in the control group. The fractured vertebrae were thoracic vertebrae in 5 and lumbar vertebrae in 11 . The number of fractured vertebrae was 1 in 14 and 2 or more in 2 . The causes of compression fracture were the appearance of low back pain in 10 , fall in 4 , and lifting a heavy object in 2 .

The muscle activity during the backward perturbation response between the CVCF and control groups is compared in Table 2. During perturbation at $4 \% \mathrm{BW}$, no significant difference was noted in the time to peak or time of onset of muscle activity. During perturbation at $6 \% \mathrm{BW}$, the time to peak anterior tibial muscle activity was significantly shorter $(P<0.05)$ and the time of onset of activity of the rectus abdominis muscle was significantly slower $(P<0.05)$ in the CVCF group than in the control group.
Table 2. Muscle activity during the backward perturbation response between the CVCF and control groups

\begin{tabular}{lccc}
\hline Variable & CVCF $(n=16)$ & Control $(n=16)$ & $P$-value \\
\hline $\begin{array}{l}\text { The time to peak activity } 4 \% B W \\
\text { TA (msec) }\end{array}$ & & \\
MV (msec) & $366.5(253.3-460.8)$ & $311.0(263.5-342.5)$ & $0.56^{b)}$ \\
RA (msec) & $268.0(208.5-648.5)$ & $299.0(266.8-380.0)$ & $0.70^{b)}$ \\
The time of onset of activity 4\%BW & & \\
TA (msec) & $170.5 \pm 56.5$ & $182.6 \pm 46.0$ & $0.51^{\text {a) }}$ \\
MV (msec) & $226.0(174.0-291.8)$ & $225.0(205.5-265.8)$ & $0.96^{b)}$ \\
RA (msec) & $238.0(198.0-275.3)$ & $275.5(248.5-361.5)$ & $0.18^{b)}$ \\
The time to peak activity 6\%BW & & \\
TA (msec) & $292.5(269.0-381.8)$ & $388.5(347.0-423.8)$ & $0.02^{b)}$ \\
MV (msec) & $378.5(336.5-412.3)$ & $428.5(354.3-448.3)$ & $0.49^{b)}$ \\
RA (msec) & $316.0(260.5-400.3)$ & $309.5(276.8-436.0)$ & $0.87^{b)}$ \\
The time of onset of activity 6\%BW & & \\
TA (msec) & $186.9 \pm 41.7$ & $161.8 \pm 37.0$ & $0.08^{a)}$ \\
MV (msec) & $247.6 \pm 64.1$ & $224.9 \pm 57.2$ & $0.30^{a)}$ \\
RA (msec) & $309.0(260.5-334.8)$ & $268.5(178.3-300.3)$ & $0.04^{b)}$ \\
\hline
\end{tabular}

Values are presented as median (interquartile range) or mean \pm standard deviation. CVCF, clinical vertebral compression fracture; $\mathrm{BW}$, body weight; $\mathrm{TA}$, tibialis anterior; $M V$, medial vastus; $R A$, rectus abdominis.

alt-test. ${ }^{\text {bl }}$ Mann-Whitney U-test.

\section{DISCUSSION}

The objective of this study was to evaluate the characteristics of muscle activity during the backward perturbation response in patients with CVCF. This study suggested that the time to peak anterior tibial muscle activity is shorter and the time of onset of rectus abdominis muscle activity is slower during the posterior perturbation response in CVCF patients.

If the floor suddenly moves forward, or the shoulder girdle or pelvic girdle is pulled in the standing position, the body sways backward or the position of the center of gravity deviates from the base of support constituted by the area of contact of the planta with the floor, possibly resulting in falling. When the body is exposed to such sudden perturbation stress, it exhibits several reactions to maintain the position of the center of gravity in the base of support. Horak and Nashner (1986) found that when backward perturbation was applied to young subjects by horizontally moving the stable floor anteriorly, synergic muscles on the anterior side of the body exhibited standard activity ascending from the anterior tibial muscle to the quadriceps femoris muscle and the rectus abdominis muscle. However, such standard muscle activity has been reported to differ depending on physical functions and characteristics, for example in older individuals compared with 
younger individuals and in older individuals with poor balance compared with those with good balance (Horak et al., 1989; Lin and Woollacott, 2002). In this study, differences in muscle activity during the backward perturbation response were observed according to the presence of CVCF.

In the CVCF and control groups, the anterior tibial, vastus medialis, and rectus abdominis muscles were activated in this order under both the $4 \% \mathrm{BW}$ and $6 \% \mathrm{BW}$ conditions. Thus, the order of the onset of activity of synergic muscles responding to backward perturbation alone suggests that both the CVCF and control groups responded to perturbation primarily by the ankle strategy. However, it has been reported that the body responds to perturbation first by the genuine ankle strategy and then by the hip strategy with increases in the strength of perturbation (Runge et al., 1999). Therefore, it is possible that not only the genuine ankle strategy but also the hip strategy was used for posture control in the subjects of this study. Through this connection, muscle activity differed between the CVCF and control groups only under the $6 \% \mathrm{BW}$ condition. In consideration of the above reports, the hip strategy is considered to have been added to the initial ankle strategy with the increase in the strength of perturbation from $4 \% \mathrm{BW}$ to $6 \% \mathrm{BW}$, which may have caused the differences in muscle activity between the two groups at $6 \% \mathrm{BW}$.

We speculate the cause of shortening of the time to peak anterior tibial muscle activity and the delay in the onset of rectus abdominis muscle activity in the CVCF group to be as follows: First, low back pain may have played a role in the delay in the onset of rectus abdominis muscle activity. According to previous studies of muscle reactions to unexpected perturbation in low back pain patients (Jacobs et al., 2016; Shenoy et al., 2013), the time of onset of the activity of trunk muscles, including the rectus abdominis muscle, was slower in low back pain patients than in no-low back pain patients. In this study, the NRS score was significantly higher in the CVCF group than in the control group, suggesting that low back pain caused a delay in the onset of rectus abdominis muscle activity.

Next, regarding the decrease in the time to peak anterior tibial muscle activity and the delay in the onset of rectus abdominis muscle activity, the vertebral compression fracture itself may have played a role. The somatic sense processes unexpected perturbation most quickly, and the body was suggested to strongly depend on somatic sense in postural control against perturbation (Dietz et al., 1994; Horak et al., 1994). Proprioception, which is one of the somatic senses, functions in the stability of the body in the environment according to sensory input from muscle spindles, tendon organs, skin receptors, and joint receptors (Andersson and Magnusson, 2002; Kavounoudias et al., 1999). In patients with lumbar vertebral disorders, spinal proprioception is reported to be reduced (Brumagne et al., 2004; Claeys et al., 2011; Johanson et al., 2011). As a result, they have been suggested to depend on sensory input from the ankle rather than the trunk and control the posture primarily by the use of muscles around the ankle (Brumagne et al., 2004). In addition, increased kyphosis may be related to shortening of the time to peak anterior tibial muscle activity. The range of spinal extension is expected to decrease with progression of kyphosis. In addition, Posterior inclination of the pelvis (relative extension of the hip) was also reported to increase with increases in kyphosis (Ota et al., 2015). In the perturbation response, the hip strategy controls the movement of the center of mass of the body by generating large and rapid motions of the hip (Horak and Nashner, 1986). For the hip strategy during the backward perturbation response, extension of the spine and hip is necessary to anteriorly shift the pelvis along the sagittal plane. However, extension of the spine and hip may become insufficient if kyphosis is increased, as in the CVCF group of this study. Thus, the CVCF group may have responded to perturbation by shortening the time to peak anterior tibial muscle activity, i.e., extensively using the ankle strategy, to compensate for the hip strategy, which was made insufficient by advanced kyphosis. Based on these observations, the shortening of the time to peak anterior tibial muscle activity and the delay in the time of onset of the rectus abdominis muscle activity in the CVCF group were speculated to have been induced by low back pain, decline in spinal proprioception, and increase in kyphosis associated with vertebral compression fracture.

Moreover, the shortening of the time to peak anterior tibial muscle activity may have been related to the delay in the time of onset of rectus abdominis muscle activity in the CVCF group. In response to posterior sway of the body in the standing position, synergic muscles act in coordination to maintain the center of pressure in the base of support (Krishnamoorthy et al., 2003). However, if some of the synergic muscles are subject to restriction, and temporal or quantitative changes occur in their activity, other synergic muscles are considered to change their activity temporally or quantitatively to compensate for the defect in postural control. Therefore, although the causative relationship is unclear, the shortening of the time to peak anterior tibial muscle activity or the delay in the onset of rectus abdominis muscle activity observed in the CVCF group may have occurred secondary to whichever preceding change. 
The limitations of this study are as follows: First, the vertebral compression fractures evaluated in this study were restricted to CVCFs. In this study, the presence of morphometric fractures was not evaluated by radiography in the control group and the possibility of their inclusion cannot be denied. Therefore, it is difficult to apply the results of this study to patients diagnosed with morphometric fractures. Next, muscle activity in response to backward perturbation was evaluated simply according to temporal indices such as the time to peak muscle activity and time of onset of muscle activity. In patients with vertebral compression fractures, exertion of maximum voluntary contraction of the trunk muscles is not recommended because it may induce re-fracture and exacerbate pain. However, explorative attempts at normalization using the peak value have been made in previous studies (Greig et al., 2014), and quantitative evaluation of muscle activity is considered necessary in the future.

As a conclusion of this study, shortening of the time to peak anterior tibial muscle activity and delay in the time to onset of rectus abdominis muscle activity were suggested during the backward perturbation response in patients with CVCF. These results suggest the necessity of developing a rehabilitation program with particular attention to neuromuscular responses to perturbation for the prevention of backward falling by CVCF patients.

\section{CONFLICT OF INTEREST}

No potential conflict of interest relevant to this article was reported.

\section{REFERENCES}

Andersson G, Magnusson M. Neck vibration causes short-latency electromyographic activation of lower leg muscles in postural reactions of the standing human. Acta Otolaryngol 2002;122:284-288.

Arima K, Abe Y, Nishimura T, Okabe T, Tomita Y, Mizukami S, Kanagae M, Aoyagi K. Association of vertebral compression fractures with physical performance measures among community-dwelling Japanese women aged 40 years and older. BMC Musculoskelet Disord 2017;18:176.

Bow $\mathrm{CH}$, Cheung E, Cheung CL, Xiao SM, Loong C, Soong C, Tan KC, Luckey MM, Cauley JA, Fujiwara S, Kung AW. Ethnic difference of clinical vertebral fracture risk. Osteoporos Int 2012;23:879-885.

Brumagne S, Cordo P, Verschueren S. Proprioceptive weighting changes in persons with low back pain and elderly persons during upright standing. Neurosci Lett 2004;366:63-66.
Chandler JM, Duncan PW, Studenski SA. Balance performance on the postural stress test: comparison of young adults, healthy elderly, and fallers. Phys Ther 1990;70:410-415.

Claeys K, Brumagne S, Dankaerts W, Kiers H, Janssens L. Decreased variability in postural control strategies in young people with non-specific low back pain is associated with altered proprioceptive reweighting. Eur J Appl Physiol 2011;111:115-123.

Crenshaw JR, Bernhardt KA, Achenbach SJ, Atkinson EJ, Khosla S, Kaufman $\mathrm{KR}$, Amin S. The circumstances, orientations, and impact locations of falls in community-dwelling older women. Arch Gerontol Geriatr 2017; 73:240-247.

Cummings SR, Black DM, Thompson DE, Applegate WB, Barrett-Connor E, Musliner TA, Palermo L, Prineas R, Rubin SM, Scott JC, Vogt T, Wallace $\mathrm{R}$, Yates AJ, LaCroix AZ. Effect of alendronate on risk of fracture in women with low bone density but without vertebral fractures: results from the Fracture Intervention Trial. JAMA 1998;280:2077-2082.

Dietz V, Schubert M, Discher M, Trippel M. Influence of visuoproprioceptive mismatch on postural adjustments. Gait Posture 1994;2:147155.

Freitas SS, Barrett-Connor E, Ensrud KE, Fink HA, Bauer DC, Cawthon PM, Lambert LC, Orwoll ES; Osteoporotic Fractures in Men (MrOS) Research Group. Rate and circumstances of clinical vertebral fractures in older men. Osteoporos Int 2008;19:615-623.

Greig AM, Briggs AM, Bennell KL, Hodges PW. Trunk muscle activity is modified in osteoporotic vertebral fracture and thoracic kyphosis with potential consequences for vertebral health. PLoS One 2014;9:e109515.

Hasserius R, Karlsson MK, Nilsson BE, Redlund-Johnell I, Johnell O; European Vertebral Osteoporosis Study. Prevalent vertebral deformities predict increased mortality and increased fracture rate in both men and women: a 10-year population-based study of 598 individuals from the Swedish cohort in the European Vertebral Osteoporosis Study. Osteoporos Int 2003;14:61-68.

Horak FB, Nashner LM. Central programming of postural movements: adaptation to altered support-surface configurations. J Neurophysiol 1986;55:1369-1381.

Horak FB, Shupert CL, Dietz V, Horstmann G. Vestibular and somatosensory contributions to responses to head and body displacements in stance. Exp Brain Res 1994;100:93-106.

Horak FB, Shupert CL, Mirka A. Components of postural dyscontrol in the elderly: a review. Neurobiol Aging 1989;10:727-738.

Ismail AA, O'Neill TW, Cooper C, Finn JD, Bhalla AK, Cannata JB, Delmas P, Falch JA, Felsch B, Hoszowski K, Johnell O, Diaz-Lopez JB, Lopez Vaz A, Marchand F, Raspe H, Reid DM, Todd C, Weber K, Woolf A, Reeve J, Silman AJ. Mortality associated with vertebral deformity in men and women: results from the European Prospective Osteoporosis 
Study (EPOS). Osteoporos Int 1998;8:291-297.

Jacobs JV, Roy CL, Hitt JR, Popov RE, Henry SM. Neural mechanisms and functional correlates of altered postural responses to perturbed standing balance with chronic low back pain. Neuroscience 2016;339:511524.

Johanson E, Brumagne S, Janssens L, Pijnenburg M, Claeys K, Pääsuke M. The effect of acute back muscle fatigue on postural control strategy in people with and without recurrent low back pain. Eur Spine J 2011;20: 2152-2159.

Kavounoudias A, Gilhodes JC, Roll R, Roll JP. From balance regulation to body orientation: two goals for muscle proprioceptive information processing? Exp Brain Res 1999;124:80-88.

Klotzbuecher CM, Ross PD, Landsman PB, Abbott TA 3rd, Berger M. Patients with prior fractures have an increased risk of future fractures: a summary of the literature and statistical synthesis. J Bone Miner Res 2000;15:721-739.

Krishnamoorthy V, Latash ML, Scholz JP, Zatsiorsky VM. Muscle synergies during shifts of the center of pressure by standing persons. Exp Brain Res 2003;152:281-292.

Lin SI, Woollacott MH. Postural muscle responses following changing balance threats in young, stable older, and unstable older adults. J Mot Behav 2002;34:37-44.

Milne JS, Lauder IJ. Age effects in kyphosis and lordosis in adults. Ann
Hum Biol 1974:1:327-337.

O'Neill TW, Cockerill W, Matthis C, Raspe HH, Lunt M, Cooper C, Banzer D, Cannata JB, Naves M, Felsch B, Felsenberg D, Janott J, Johnell O, Kanis JA, Kragl G, Lopes Vaz A, Lyritis G, Masaryk P, Poor G, Reid DM, Reisinger W, Scheidt-Nave C, Stepan JJ, Todd CJ, Woolf AD, Reeve J, Silman AJ. Back pain, disability, and radiographic vertebral fracture in European women: a prospective study. Osteoporos Int 2004;15:760765 .

Ota S, Goto H, Noda Y, Fujita R, Matsui Y. Relationship between standing postural alignments and physical function among elderly women using day service centers in Japan. J Back Musculoskelet Rehabil 2015; 28:111-117.

Runge CF, Shupert CL, Horak FB, Zajac FE. Ankle and hip postural strategies defined by joint torques. Gait Posture 1999;10:161-170.

Shenoy S, Balachander H, Sandhu JS. Long latency reflex response of superficial trunk musculature in athletes with chronic low back pain. J Back Musculoskelet Rehabil 2013;26:445-450.

Stone KL, Seeley DG, Lui LY, Cauley JA, Ensrud K, Browner WS, Nevitt MC, Cummings SR; Osteoporotic Fractures Research Group. BMD at multiple sites and risk of fracture of multiple types: long-term results from the Study of Osteoporotic Fractures. J Bone Miner Res 2003;18: 1947-1954. 